\title{
Consistencia interna y validez concurrente del cuestionario para evaluación de limitaciones y restricciones en niños con TDAH
}

Internal Consistency and Concurrent Validity of the Questionnaire for Limitations and Restrictions Assessment in Children with ADHD

Consistência interna e validez concorrente do questionário para avaliação de limitações e restrições em crianças com TDAH Luisa Matilde Salamanca-Duque MsC, 'María Mercedes del Carmen Naranjo-Aristizábal MsC,' Mónica Méndez-Narváez $\mathrm{Ft}^{2}$ Diana Patricia Sánchez $\mathrm{Ft}^{3}$

Recibido: 7 de noviembre de $2013 \bullet$ Aceptado: 1 de abril de 2014

Doi: dx.doi.org/10.12804/revsalud12.03.2014.06

Para citar este artículo: Salamanca-Duque LM, Naranio-Aristizábal MMC, Méndez-Narváez M, Sánchez DP. Consistencia interna y validez concurrente del cuestionario para evaluación de limitaciones y restricciones en niños con TDAH. Rev Cienc Salud. 2014;12 (3): 371-84 . doi: dx.doi.org/10.12804/revsalud12.03.2014.06

\section{Resumen}

Introducción: El TDAH es uno de los diagnósticos más frecuentes en psiquiatría infantil, su diagnóstico temprano reviste una gran importancia para su intervención familiar, escolar y social. Basándose en la clasificación internacional del funcionamiento, la discapacidad y la salud CIF, se diseñó un cuestionario para la evaluación de limitaciones en la actividad y restricciones en la participación en niños con TDAH, el cuestionario CLARP-TDAH versión padres y profesores. Objetivo: Determinar el grado de consistencia interna de los CLARP-TDAH y su validez concurrente con el cuestionario de capacidades y dificultades SDQ versión padres y maestros. Materiales y métodos: Muestra de 203 niños de 6 a 12 años con TDAH, escolarizados, en cinco ciudades de Colombia. Se aplicaron estos cuestionarios a padres y profesores. El análisis de consistencia interna se realizó por coeficiente de Cronbach, y la validez concurrente mediante coeficiente de correlación de Spearman y con predictores múltiples y únicos a través de modelos de regresión lineal múltiple y simple. Resultados: La consistencia interna fue elevada para los cuestionarios globales y para cada uno de sus dominios. El CLARP-TDAH padres obtuvo consistencia interna

\footnotetext{
1 Departamento Movimiento Humano, Universidad Autónoma de Manizales. Correspondencia: Iuisasalamanca@autonoma.edu.co

2 Fisiocenter, Popayán.

3 Universidad Santiago de Cali Seccional Palmira.
} 
de 0,83 y el CLARP-TDAH profesores de 0,93. Se halló validez concurrente entre los CLARPTDAH y el SDQ padres y maestros, existe concurrencia entre el CLARP-TDAH profesores y SDQ maestros, y entre el CLARP-TDAH padres y profesores, esto dado por valores $\mathrm{p}<0,001$.

Palabras clave: Trastorno por déficit de atención con hiperactividad (TDAH), Clasificación Internacional del Funcionamiento, de la Discapacidad y de la Salud (CIF), reproducibilidad de resultados, validez de las pruebas.

\section{Abstract}

Introduction: ADHD is one of the most common diagnoses in child psychiatry, its early diagnosis is of great importance for intervention at family, school and social environment. Based on the International Classification of Functioning, Disability and Health (ICF), a questionnaire was designed to assess activity limitations and participation restrictions in children with ADHD. The questionnaire was called "CLARP-ADHD Parent and Teacher Version". Objective: To determine the degree of internal consistency of the CLARP-ADHD questionnaire, and its concurrent validity with the "Strengths and Difficulties Questionnaire SDQ parent and teacher version". Material and Methods: A sample of 203 children aged 6 to 12 with ADHD, currently attending school in five Colombian cities. The questionnaires were applied to parents and teachers. The internal consistency analysis was performed through Cronbach coefficient and concurrent validity using the Spearman correlation coefficient utilizing multiple and unique predictors through multiple linear regression as well as simple regression models. Results: A high internal consistency was found for global questionnaires for each of its domains. The CLARP-ADHD for parents gave as result an internal consistency of 0.83 , and the CLARP-ADHD for teachers one of 0.93 . Concurrent validity was found between the CLARP-ADHD and the SDQ Parent and Teacher version; also, concurrence between the CLARPADHD for Teachers and the SDQ Teachers was found, as well as between CLARP ADHD for Parents and CLARP ADHD Teachers, given by $\mathrm{p}$ values of $\mathrm{p}<0.001$.

Key Words: Attention Deficit and Hyperactivity Disorder (ADHD), International Classification of Functioning, Disability and Health (ICF), Results Reproducibility, Test Validity.

\section{Resumo}

Introdução: OTDAH é um dos diagnósticos mais frequentes em psiquiatria infantil, seu diagnóstico precoce reviste uma grande importância para sua intervenção no nível familiar, escola e social. Baseando-se na classificação internacional do funcionamento, a deficiência e a saúde CIF, criou-se um questionário para a avaliação de limitações na atividade e restrições na participação em crianças com TDAH, o questionário CLARP-TDAH Versão Pais e Professores. Objetivo: Determinar o grau de consistência interna dos CLARP TDAH e sua validez concorrente com o questionário de capacidades e dificuldades SDQ versão pais e professores. Materiais e Métodos: Amostra de 203 crianças de 6 e 12 anos com TDAH, escolarizados, em cinco cidades da Colômbia. Aplicaram-se estes questionários a pais e professores. A análise de consistência interna realizou-se por coeficiente de Cronbach, e a validez concorrente mediante coeficiente de correlação de Spearman e com preditores múltiplos e únicos através de modelos de regressão lineal múltipla e simples. 
Resultados: A consistência interna foi elevada para os questionários globais e para cada um de seus domínios. O questionário CLARP-TDAH dos pais obteve consistência interna de 0,83, e o CLARP-TDAH dos professores de 0,93. Achou-se validez concorrente entre os CLARP TDAH e o SDQ Pais e Professores, existe concorrência entre o CLARP TDAH Professores e SDQ Professores, e entre o CLARP TDAH Pais e CLARP TDAH Professores, isto dado por valores $\mathrm{p}<0,001$.

Palavras-chave: Transtorno do Déficit de Atenção com Hiperatividade TDAH, Classificação Internacional do Funcionamento, da Deficiência e da Saúde CIF, reprodutibilidade de resultados, validez das provas.

\section{Introducción}

El trastorno por déficit de atención con hiperactividad TDAH se define como un síndrome neurocomportamental frecuente, caracterizado por hiperactividad, impulsividad e inatención, las cuales afectan el desarrollo integral del niño (1). Es uno de los problemas de comportamiento de índole crónico más común en la infancia, especialmente en el período escolar y representa un problema complejo, debido a que aparece en edades tempranas, repercute en la vida diaria del niño desde todas las esferas del desarrollo y existe la probabilidad de que persista a lo largo de la vida (2-5).

La Asociación Americana de Psiquiatría (APA) identifica tres síntomas nucleares, inatención, hiperactividad e impulsividad, pero además se pueden sumar otras manifestaciones asociadas como comorbilidad. Estas dificultades llevan a los niños a continuas experiencias de fracaso, las cuales pueden ser más generalizadas en el ámbito familiar, educativo y social, y se convierten en limitaciones en la actividad diaria funcional y en restricciones en la participación social que afectan el funcionamiento personal $\mathrm{y}$ en sociedad.

El TDAH ha sido abordado para su diagnóstico básicamente desde la clínica, con un mayor énfasis en el aspecto biológico y en consecuencias de la enfermedad desde las clasificaciones CIE-10, DSM-IV y fenómenos de comorbilidad, los cuales no están enmarcados directamente en la conceptualización actual del modelo biopsicosocial de la Clasificación Internacional del Funcionamiento, la Discapacidad y la Salud (CIF) de la Organización Mundial de la Salud (OMS), bajo el cual se deben mirar las condiciones de salud (6-8).

La CIF describe el funcionamiento humano como un término genérico que incluye funciones y estructuras corporales, actividades y participación social, indicando los aspectos positivos de la interacción de un individuo en una condición de salud específica con los factores contextuales ambientales y personales que lo determinan. Desde esta perspectiva, CIE y CIF son complementarias y en combinación posibilitan un mejor análisis a la situación de los niños y las niñas con TDAH.

En este sentido, en Colombia, desde el año 2009, se precisó un esfuerzo para diseñar un instrumento válido y confiable que permitiera valorar el grado de interferencia en el funcionamiento adaptativo del niño con TDAH en diferentes contextos, especialmente familiar y escolar, trascendiendo la intervención hacia perspectivas más amplias de la salud, el desarrollo infantil y sus diferentes componentes. Dicho instrumento es el nominado 'Cuestionario para la evaluación de limitaciones en la actividad y restricciones en la participación en niños con TDAH, CLARP-TDAH versión padres y CLARP-TDAH versión profesores', los 
cuales permiten caracterizar el funcionamiento de los niños y las niñas en los contextos familiar y escolar según el desempeño reportado por los padres y los profesores en los diferentes dominios considerados por la CIF, como aprendizaje y aplicación del conocimiento, tareas y demandas generales, autocuidado, movilidad, interacciones y relaciones interpersonales, entre otros. Un primer estudio realizado con estos cuestionarios en la ciudad de Manizales, Colombia, con una muestra de 42 niños y niñas de 6 a 12 años de edad con TDAH, demostró un adecuado nivel de confiabilidad por consistencia interna, dado por un coeficiente Alfa de Cronbach de 0,87 para el CLARP-TDAH padres y de 0,96 para el CLARP-TDAH profesores $(9,10)$. Considerando la necesidad de obtener dicho nivel de confiabilidad en una población mayor, el presente estudio tuvo como objetivo determinar nuevamente su confiabilidad por consistencia interna en una muestra de niños y niñas mayor en algunas ciudades colombianas y su validez concurrente con el Cuestionario de Capacidades y Dificultades (SDQ) versión padres y maestros (11).

\section{Materiales y métodos}

El estudio se ubicó en un enfoque empíricoanalítico orientado específicamente hacia la validación de un instrumento. La muestra se obtuvo de manera no probabilística a conveniencia y estuvo constituida por 203 niños y niñas de 6 a 12 años de edad, escolarizados, con diagnóstico de TDAH confirmado a través de la entrevista Minikid, quienes fueron reclutados a través de instituciones de apoyo terapéutico de las ciudades de Bogotá, Cali, Popayán, Cartagena y Bucaramanga. Al obtener la lista de los niños de las instituciones de apoyo terapéutico se realizó contacto telefónico con sus padres y, una vez autorizaron la participación en el estu- dio, se llevó a cabo la firma de consentimiento informado.

En el estudio, la técnica fue la entrevista personal con uno de los padres y el profesor correspondiente a su grado escolar, y los instrumentos fueron el CLARP-TDAH padres, el CLARP-TDAH profesores, el SDQ padres y el SDQ maestros.

\section{Instrumentos}

CLARP-TDAH versión padres y profesores

Ambos cuestionarios permiten evaluar limitaciones en la actividad y restricciones en la participación determinando un perfil de funcionamiento en términos de dificultades en la realización de diversas actividades familiares y escolares de la vida diaria. El cuestionario CLARP-TDAH padres está constituido por 14 ítems, distribuidos en 2 dominios que incluyen: tareas y demandas generales con 3 ítems y autocuidado con 11 ítems. El cuestionario CLARPTDAH profesores está constituido por 20 ítems distribuidos en 4 dominios que incluyen: aprendizaje, tareas y demandas generales con 7 ítems, movilidad con 7 ítems, interacciones y relaciones interpersonales con 3 ítems, y vida comunitaria, social y cívica con 3 ítems. Ambos cuestionarios contienen preguntas que califican el grado de dificultad de los niños para la realización de actividades familiares y escolares, calificando en una escala de 0 a 4 conforme a lo establecido por la CIF así: 0 ninguna dificultad, 1 dificultad ligera, 2 dificultad moderada, 3 dificultad grave, 4 dificultad completa. El tiempo determinado para responder los cuestionarios CLARP-TDAH padres y CLARP-TDAH profesores fue aproximadamente de 15 minutos. La puntuación final por cada instrumento en una escala de 0 a 100 llega a interpretarse tal como lo propone la CIF: ninguna dificultad (0-4\%), dificultad ligera (5$24 \%$ ), dificultad moderada (25-49\%), dificultad grave (50-95\%), dificultad completa (96-100\%). 
Cuestionario de capacidades y dificultades, $S D Q$ versión padres y maestros

El SDQ es un cuestionario que tiene por objetivo investigar los problemas conductuales de los pequeños entre los 4 y 16 años de edad (11); es un breve cuestionario que indaga sobre las capacidades y dificultades en 25 ítems distribuidos en cinco componentes: problemas de conducta, hiperactividad, síntomas emocionales, problemas con compañeros/as y conducta prosocial. El cuestionario debe ser completado por padres o maestros. Para cada una de los cinco componentes, la puntuación puede variar desde 0 hasta 10 .

En el estudio se consideró relevante realizar una validez concurrente entre ambos instrumentos puesto que el SDQ es un cuestionario que permite caracterizar el comportamiento en niños principalmente con desórdenes de tipo hiperactividad e inatención (12-14).

El análisis de la información se realizó mediante software estadístico SPSS versión 19.0, a través de análisis descriptivo univariado y bivariado aplicando pruebas estadísticas tales como coeficiente de correlación de Spearman (Rho) con predictores múltiples y únicos a través de modelos de regresión lineal múltiple y simple, respectivamente, y con la aplicación del coeficiente Alfa de Cronbach para determinar la consistencia interna.

El Rho se aplica para variables de tipo ordinal que permite obtener valores entre -1 y 1 , en general se asumen como índices que demuestran correlación desde el valor 0,2 , considerando que entre 0,2-0,4 corresponde a una correlación baja; 0,4-0,6 correlación moderada; y mayores a 0,6 a una correlación alta, con un nivel de confianza del 95\% y un $\mathrm{p}<0,05$ evidenciando significancia estadística. Respecto al coeficiente alfa de Cronbach este puede oscilar entre 0 y 1 y se considera una adecuada consistencia interna valores superiores a 0,7 , e idealmente entre 0,8 y 0,9.

Según la declaración de Helsinki y la Resolución 008430 del Ministerio de Salud Nacional de Colombia este estudio se clasificó como investigación sin riesgo. El estudio fue aprobado por el comité de bioética de la Universidad Autónoma de Manizales registrado en el Acta \# 020 de 2011.

\section{Resultados}

\section{Caracterización sociodemográfica}

Respecto a la caracterización sociodemográfica de los participantes del estudio, se encontró que el $66,5 \%$ de los niños fueron de género masculino y el 33,5 \% del género femenino. La edad de los participantes osciló en un rango de 6 a 12 años, promedio $8,6 \pm 1,7$. El 75,4\% pertenecía a estratos considerados como socioeconómicamente bajos y medios bajos. Los padres de familia reportaron que los niños con TDAH en un $29,1 \%$ recibían tratamiento farmacológico y el $81,8 \%$ estaban involucrados en algún programa de apoyo terapéutico.

\section{Consistencia interna}

El cuestionario CLARP-TDAH padres obtuvo un coeficiente Alfa de Cronbach de 0,88 para el cuestionario completo y valores adecuados para cado uno de sus dominios. También se realizó el análisis ante la eliminación de cada uno de los ítems, encontrándose que la consistencia interna del mismo no se modifica ante la eliminación de los ítems aislados (tabla 1). El cuestionario CLARP-TDAH profesores obtuvo un valor Alfa de Cronbach de 0,93 lo que evidencia una consistencia interna alta, y cada uno de sus dominios valores entre 0,81 y 0,89. Ante la eliminación de cada uno de los ítems no se considera cambio en la consistencia interna (tabla 2). 
Tabla 1. Valores Alfa de Cronbach CLARP TDAH Padres

\begin{tabular}{|c|c|c|c|c|}
\hline $\begin{array}{l}\text { Dominio del } \\
\text { cuestionario }\end{array}$ & $\begin{array}{l}\text { Alfa de cronbach } \\
\qquad(N=203)\end{array}$ & Items del dominio & $\begin{array}{l}\text { Correlación item - } \\
\text { cuestionario }\end{array}$ & $\begin{array}{l}\text { Alfa de cronbach si } \\
\text { se elimina el ítem }\end{array}$ \\
\hline \multirow{3}{*}{$\begin{array}{l}\text { Tareas y } \\
\text { demandas } \\
\text { generales }\end{array}$} & \multirow{3}{*}{0,681} & Completar la rutina diaria & 0,411 & 0,879 \\
\hline & & Dirigir el propio nivel de actividad & 0,311 & 0,883 \\
\hline & & Manejo del estrés & 0,433 & 0,879 \\
\hline \multirow{11}{*}{ Autocuidado } & \multirow{11}{*}{0,888} & Lavar partes individuales del cuerpo & 0,678 & 0,866 \\
\hline & & Lavar todo el cuerpo & 0,665 & 0,866 \\
\hline & & Secarse & 0,650 & 0,867 \\
\hline & & Cuidado de los dientes & 0,598 & 0,870 \\
\hline & & Cuidado del pelo & 0,618 & 0,869 \\
\hline & & Regulación de la micción & 0,441 & 0,877 \\
\hline & & Regulación de la defecación & 0,409 & 0,878 \\
\hline & & Ponerse la ropa & 0,714 & 0,864 \\
\hline & & Quitarse la ropa & 0,633 & 0,868 \\
\hline & & Comer & 0,515 & 0,874 \\
\hline & & Beber & 0,628 & 0,870 \\
\hline
\end{tabular}

Tabla 2. Valores Alfa de Cronbach CLARP TDAH Profesores

\begin{tabular}{|c|c|c|c|c|}
\hline $\begin{array}{l}\text { Dominio del } \\
\text { cuestionario }\end{array}$ & $\begin{array}{l}\text { Alfa de cronbach } \\
\qquad(\mathrm{N}=203)\end{array}$ & Items del dominio & $\begin{array}{c}\text { Correlación item - } \\
\text { cuestionario }\end{array}$ & $\begin{array}{l}\text { Alfa de cronbach si } \\
\text { se elimina el ítem }\end{array}$ \\
\hline \multirow{7}{*}{$\begin{array}{l}\text { Aprendizaje, tareas y } \\
\text { demandas generales }\end{array}$} & \multirow{7}{*}{0,89} & Centrar la atención & 0,581 & 0,93 \\
\hline & & Leer & 0,622 & 0,929 \\
\hline & & Escribir & 0,675 & 0,928 \\
\hline & & Calcular & 0,721 & 0,927 \\
\hline & & Resolver problemas & 0,694 & 0,928 \\
\hline & & $\begin{array}{l}\text { Llevar a cabo una única tarea } \\
\text { independientemente }\end{array}$ & 0,613 & 0,929 \\
\hline & & Manejo de responsabilidades & 0,688 & 0,928 \\
\hline \multirow{7}{*}{ Movilidad } & \multirow{7}{*}{0,81} & Permanecer sentado & 0,444 & 0,933 \\
\hline & & Manipular & 0,678 & 0,928 \\
\hline & & Lanzar & 0,344 & 0,938 \\
\hline & & Atrapar & 0,615 & 0,929 \\
\hline & & Andar sorteando obstáculos & 0,654 & 0,929 \\
\hline & & Correr & 0,616 & 0,930 \\
\hline & & Saltar & 0,617 & 0,930 \\
\hline \multirow{3}{*}{$\begin{array}{l}\text { Interacciones } \\
\text { y relaciones } \\
\text { interpersonales }\end{array}$} & \multirow{3}{*}{0,84} & Establecer relaciones & 0,695 & 0,928 \\
\hline & & $\begin{array}{l}\text { Regulación del } \\
\text { comportamiento en las } \\
\text { interacciones }\end{array}$ & 0,653 & 0,929 \\
\hline & & Tolerancia en las relaciones & 0,597 & 0,930 \\
\hline \multirow{3}{*}{$\begin{array}{l}\text { Vida comunitaria social } \\
\text { y cívica }\end{array}$} & \multirow{3}{*}{0,87} & Juego & 0,716 & 0,966 \\
\hline & & Deportes & 0,734 & 0,966 \\
\hline & & Manualidades & 0,705 & 0,966 \\
\hline
\end{tabular}


Validez concurrente

La validez concurrente se obtuvo a través de la comparación entre los resultados del CLARPTDAH padres y profesores dado en términos de grado de dificultad en actividades y participación, y del SDQ padres y maestros dado en términos de dificultad en problemas de conducta, teniendo en cuenta que todos estos constructos se relacionan con el desempeño de los niños en su vida cotidiana familiar y escolar. Para determinar esta validez, inicialmente se realizaron las pruebas de normalidad de cada una de las variables a través de la prueba Kolmogorov-Smirnoff, encontrándose una distribución normal de las variables del SDQ profesores ( $p>0,05)$, mientras que las variables CLARP padres y profesores y SDQ padres no presentaron una distribución normal $(\mathrm{p}<0,05)$ (tabla 3). Debido a la no distribución normal se procedió a la aplicación de pruebas no paramétricas y al análisis de correlación bivariada, utilizando el coeficiente de correlación de Spearman (Rho). Se consideró una relación adecuada cuando el $p$ valor fue $\leq 0,05$, interpretando el grado de correlación entre 0 y 1 . Los resultados obtenidos para determinar la validez concurrente evidenciaron correlación entre CLARP-TDAH padres y profesores y entre CLARP-TDAH profesores y SDQ maestros, por valores Rho superiores a 0,37 y $\mathrm{p}=0,00$ (tabla 4 ).

Posteriormente, se realizó la evaluación y el análisis de concurrencia con predictores múltiples y únicos a través de modelos de regresión lineal múltiple y simple, respectivamente. En los modelos de regresión lineal se evaluó la significancia individual de los betas y la prueba global de los modelos; igualmente, se estableció el coeficiente de validez a través del $R$, considerando significancia con un $\mathrm{p} \leq 0,05$.

Tabla 3. Prueba de normalidad para los resultados totales de CLARP TDAH Padres, CLARP TDAH Profesores, SDQ Padres y SDQ Maestros (Prueba K-S)

\begin{tabular}{llcccc}
\hline & & $\begin{array}{c}\text { CLARP TDAH } \\
\text { profesores }\end{array}$ & $\begin{array}{c}\text { CLARP TDAH } \\
\text { padres }\end{array}$ & $\begin{array}{c}\text { SDQ } \\
\text { padres }\end{array}$ & $\begin{array}{c}\text { SDQ } \\
\text { maestros }\end{array}$ \\
\hline $\mathrm{N}$ & & 203 & 203 & 203 & 203 \\
\hline \multirow{2}{*}{ Parámetros normales } & Media & 33,42 & 28,31 & 20,67 & 19,02 \\
& Desviación típica & 17,847 & 16,328 & 4,901 & 4,613 \\
\hline \multirow{2}{*}{$\begin{array}{l}\text { Diferencias más } \\
\text { extremas }\end{array}$} & Absoluta & 0,110 & 0,127 & 0,111 & 0,079 \\
& Positiva & 0,110 & 0,127 & 0,111 & 0,079 \\
\hline Z de Kolmogorov-Smirnov & Negativa & $-0,066$ & $-0,073$ & $-0,052$ & $-0,052$ \\
Sig. asintót. (bilateral) & & 1,566 & 1,810 & 1,576 & 1,120 \\
\hline
\end{tabular}

Tabla 4. Correlación de medidas entre los instrumentos CLARP TDAH y SDQ

\begin{tabular}{lcc}
\hline \multicolumn{1}{c}{ Correlación de instrumentos } & Rho & P valor \\
\hline CLARP TDAH padres Vs CLARP TDAH profesores & 0,41 & 0,00 \\
CLARP TDAH padres Vs SDQ padres & 0,11 & 0,11 \\
CLARP TDAH profesores Vs SDQ maestros & 0,37 & 0,00 \\
\hline
\end{tabular}


Tabla 5. Significancia individual de los beta del modelo de regresión lineal múltiple

\begin{tabular}{lcccccc}
\hline \multicolumn{1}{c}{$\begin{array}{c}\text { Modelo } 1 \\
\text { (CLARP TDAH Profesores Vs CLARP } \\
\text { TDAH Padres y SDQ Maestros) }\end{array}$} & \multicolumn{2}{c}{$\begin{array}{c}\text { Coeficientes no } \\
\text { estandarizados }\end{array}$} & $\begin{array}{c}\text { Coeficientes } \\
\text { tipificados }\end{array}$ & T & Sig. \\
\cline { 2 - 5 } (Constante) & B & Error típico & Beta & & & 0,410 \\
CLARP TDAH padres & 0,948 & 4,783 & & & 0,825 & 0,000 \\
SDQ maestros & 0,439 & 0,068 & 0,402 & & 6,453 & 0,000 \\
\hline
\end{tabular}

Tabla 6. Coeficiente de validez y prueba global del modelo de regresión lineal múltiple

\begin{tabular}{|c|c|c|}
\hline \multicolumn{2}{|c|}{ Prueba } & \multirow{2}{*}{$\begin{array}{l}\text { Valor } \\
0,497\end{array}$} \\
\hline \multirow{4}{*}{ Resumen del modelo 1} & $\mathrm{R}$ & \\
\hline & $\mathrm{R}$ cuadrado & 0,247 \\
\hline & R cuadrado corregida & 0,240 \\
\hline & Error típico de la estimación & 15,563 \\
\hline \multirow{2}{*}{ Prueba global del modelo 1 (ANOVA) } & $\mathrm{F}$ & 32,825 \\
\hline & Significancia & 0,00 \\
\hline
\end{tabular}

Validez concurrente con múltiples predictores, modelo de regresión lineal múltiple Dada la correlación positiva entre el CLARPTDAH profesores con el CLARP-TDAH padres y el SDQ maestros se encuentra la oportunidad de realizar este modelo, en el que se determinó como variable dependiente el CLARP-TDAH profesores y variables predictivas el CLARPTDAH padres y el SDQ maestros. Se realizó exploración del modelo de regresión lineal múltiple, a través del modelo predictivo constituido por las variables CLARP-TDAH padres y SDQ maestros (tabla 5).

Posteriormente, se realizó el análisis del coeficiente de validez para el criterio del conjunto de predictores y la prueba global del modelo, y se encontró un adecuado coeficiente de validez, casi un $50 \%$, lo que significa que los resultados de los cuestionarios predictores CLARP-TDAH padres y SDQ maestros explican en un $25 \%$ las limitaciones y restricciones dadas por el CLARP-TDAH profesores, con esto se evi- dencia un moderado nivel de concurrencia de este instrumento desde el modelo de regresión múltiple (tabla 6).

Validez concurrente con predictores únicos, modelos de regresión lineal simple

Se determinaron otros tres modelos; en dos de ellos, el modelo 2 y el modelo 3 , se tuvo como variable dependiente el CLARP-TDAH profesores y como variables predictivas el SDQ maestros y el CLARP-TDAH padres, respectivamente. El modelo 4 con variable dependiente el CLARP padres y como variable predictiva el CLARP-TDAH profesores.

Finalmente, los modelos de regresión lineal simple para los instrumentos predictores evidencian validez concurrente con el CLARP-TDAH profesores por un $\mathrm{p}<0,001$, y con el CLARPTDAH padres por un $\mathrm{p}<0,001$, sin embargo cabe reconocer que el coeficiente de validez $\mathrm{R}^{2}$ resultó solamente entre un $9 \%$ para el modelo 2 y un $19 \%$ para los modelos 3 y 4 (tabla 7 ). 
Tabla 7. Modelos de regresión lineal simple

\begin{tabular}{|c|c|c|c|c|c|c|c|}
\hline \multirow[b]{2}{*}{ Modelos } & \multicolumn{3}{|c|}{ Resumen del modelo } & \multicolumn{2}{|c|}{$\begin{array}{l}\text { Prueba global del } \\
\text { modelo (ANOVA) }\end{array}$} & \multicolumn{2}{|c|}{$\begin{array}{l}\text { Significancia } \\
\text { de los BETA }\end{array}$} \\
\hline & $\mathrm{R}$ & $\begin{array}{c}\mathrm{R} 2 \\
\text { corregida }\end{array}$ & $\begin{array}{l}\text { Error } \\
\text { típico } \\
\text { estimado }\end{array}$ & $\mathrm{F}$ & Sig & Beta & Sig \\
\hline $\begin{array}{l}\text { Modelo 2: CLARP TDAH } \\
\text { profesores Vs SDQ } \\
\text { profesores }\end{array}$ & 0,301 & 0,086 & 17,064 & 19,967 & 0,000 & 1,163 & 0,000 \\
\hline $\begin{array}{l}\text { Modelo 3: CLARP TDAH } \\
\text { profesores Vs CLARP } \\
\text { TDAH padres }\end{array}$ & 0,442 & 0,191 & 16,051 & 48,734 & 0,000 & 0,483 & 0,000 \\
\hline $\begin{array}{l}\text { Modelo 4: CLARP TDAH } \\
\text { padres Vs CLARP TDAH } \\
\text { profesores }\end{array}$ & 0,442 & 0,191 & 14,685 & 48,734 & 0,000 & 0,404 & 0,000 \\
\hline
\end{tabular}

El resultado final de validez concurrente determina que los instrumentos CLARP-TDAH padres y profesores para la evaluación de limitaciones en la actividad y restricciones en la participación tienen validez concurrente, específicamente el CLARP-TDAH profesores desde un modelo predictivo múltiple con el SDQ maestros y el CLARP-TDAH padres, y este último desde un modelo predictivo único con el CLARP profesores. Dichas concurrencias si bien no son muy altas desde el valor $\mathrm{R}^{2}$ para todos los modelos anteriormente descritos, tienen una significancia menor a 0,001 .

Cabe resaltar que el estudio se realizó sin limitaciones que obstaculizaran la recolección de información y el análisis de resultados.

\section{Discusión}

Si bien el compromiso desde signos y síntomas es importante en el TDAH, lo es más el compromiso funcional, lo que genera mayores repercusiones en el desempeño de diferentes actividades de la vida diaria familiar, escolar y social y, por ende, necesidad de intervenciones que generan mayores costos a nivel personal, económico y social, como la desintegración familiar, el aumento en la tasa de deserción escolar o la repetición de grados escolares. Por lo tanto, es clara la importancia de llevar a cabo procesos de valoración desde la funcionalidad y el funcionamiento en niños y niñas con TDAH, que permitan evidenciar el impacto en los diferentes contextos familiar y escolar, especialmente respecto a resultados a largo plazo y no solamente a los síntomas a corto plazo $(15,16)$. Frente a esta perspectiva fueron creados los cuestionarios CLARP-TDAH padres y profesores con base en el modelo biopsicosocial de la CIF, los cuales permiten determinar un perfil de funcionamiento identificando dificultades en el desempeño de actividades relacionadas con la vida en el hogar y en la escuela, bajo dominios como aprendizaje, tareas y demandas generales, autocuidado, movilidad, interacciones y relaciones interpersonales, entre otros.

Con este estudio se logra dar un paso respecto a la evaluación de la funcionalidad y el funcionamiento de los niños y las niñas con TDAH, con lo que se obtienen unos instrumentos válidos y confiables basados en la Clasificación Internacional del Funcionamiento, la Discapacidad y la Salud (CIF) de la OMS y permiten trascender a las consecuencias personales, familiares, de amistad, escolares y de 
vida social en la población con este diagnóstico $(15,16)$. En el presente estudio se determinó la confiabilidad por consistencia interna y la validez concurrente de los cuestionarios CLARPTDAH padres y profesores en una muestra de 203 niños y niñas de cinco ciudades colombianas: Bogotá, Bucaramanga, Cali, Popayán y Cartagena.

Respecto a la consistencia interna se obtuvieron valores positivos por encima de 0,8 para los dos cuestionarios a través del coeficiente alfa de Cronbach, 0,8 para el CLARP-TDAH padres y más de 0,9 para el de profesores; igualmente, para cada uno de los dominios de los dos cuestionarios se encontraron valores positivos por encima de 0,7 , especialmente en el de profesores. Tales resultados podrían estar relacionados con conceptos descritos anteriormente, como el bajo número de ítems y la diferencia cuantitativa y cualitativa de la información de padres y profesores en los niños con TDAH (17). Estos resultados de consistencia interna diferentes entre padres y profesores también se han encontrado en otros estudios como uno realizado en Manizales, donde se encontró un alfa para el Conners de padres de 0,87 y para el Conners de profesores de 0,92, donde se reconoce igualmente redundancia en este último (18).

Respecto a los resultados de consistencia interna adecuados de ambos cuestionarios cabe citar también otras escalas aplicadas en Colombia para la valoración de niños y niñas con TDAH, como el cuestionario breve para el diagnóstico de deficiencia atencional Checklist para DDA versión colombiana, cuyos valores alfa de Cronbach para el rango de edad de 6 a 11 años oscilan entre 0,79 y 0,9 para sus subescalas, y es de 0,92 para la escala completa, y el sistema multidimensional para la evaluación de los comportamientos de los niños -Behavior Assessment System for Children (BASC) - , cuya consistencia interna en la escala clínica de hiperactividad, agresión, problemas de conducta y problemas de atención entre otros es de 0,85 y en la escala de adaptabilidad y habilidades sociales es de 0,75 (18). Para este caso, en los cuestionarios hay buena confiabilidad para asignar calificadores de desempeño de los componentes de actividad y participación de la CIF para niños con TDAH tipo combinado en rango de edad de 6 a 12 años.

La validez concurrente se analizó con respecto a los cuestionarios SDQ padres y maestros, estos instrumentos predicen dificultades desde tres grupos: desórdenes de conductas oposicionistas, de hiperactividad-inatención y de ansiedad-depresión, son instrumentos que cuentan con fuertes propiedades psicométricas, especialmente respecto al TDAH, de allí que haya sido considerado en este estudio para medir la validez concurrente con los CLARPTDAH que determinan el grado de dificultad en limitaciones en la actividad y restricciones en la participación en niños con TDAH en diferentes ámbitos y dominios de la vida diaria y escolar (12-14).

Los resultados hallados en este estudio demuestran concurrencia de los cuestionarios CLARP-TDAH para la evaluación de limitaciones en la actividad y restricciones en la participación, específicamente el CLARP-TDAH profesores con el SDQ maestros y el CLARPTDAH padres por las relaciones significativas y el coeficiente de validez para el conjunto de predictores en el modelo de regresión lineal múltiple, y el CLARP-TDAH padres con el de profesores desde el modelo de regresión lineal simple.

Es relevante reconocer que en cuanto a las correlaciones de los dominios de actividad y participación de la CIF con diferentes aspectos del desempeño de los niños, otros estudios han demostrado una fuerte y significativa correlación, entre ellos el de Battaglia y cols., donde los valores de correlación de Pearson oscilaron 
entre $r=-0,71$ y $r=-0,89$, principalmente para los dominios de aprendizaje y aplicación de conocimiento e interacciones y relaciones interpersonales, los cuales se consideran en el CLARP-TDAH profesores que fue el cuestionario que obtuvo mejor validez de concurrencia en los resultados (19). Respecto a esta concurrencia mayor en el cuestionario de profesores, cabe citar resultados de estudios del SDQ desde su validez concurrente con otras escalas, por ejemplo, con la escala Rutter, obtuvo resultados positivos, para padres $\mathrm{r}=0,88$ y profesores $\mathrm{r}=$ 0,92, con el Child Behavior Check List (CBL) tuvo valores para la subescala de problemas de conducta: 0,71 para padres y 0,79 para profesores; hiperactividad de 0,69 para padres y 0,77 para profesores; y problemas con pares de 0,52 para padres y 0,57 para profesores (13). Tales afirmaciones resaltan que es un instrumento fuerte en cualidades psicométricas, especialmente en relación a información dada por profesores, por ello se constituye en herramienta adecuada para correlacionar con el CLARPTDAH, que si bien no predice ni diagnostica el trastorno como tal, si permite caracterizar las condiciones de funcionamiento y desempeño derivadas del mismo.

En este sentido, es de reconocer que si bien existe concurrencia entre los instrumentos CLARP-TDAH profesores con SDQ profesores, y CLARP-TDAH padres con CLARP-TDAH profesores, su valor $\mathrm{R} 2$ no fue suficientemente significativo, es posible que esto esté dado porque los instrumentos CLARP-TDAH tienen un constructo predominante en relación a actividades y participación social de los niños en su cotidianidad desde múltiples áreas como el aprendizaje, las tareas y demandas generales, comunicación, autocuidado, movilidad, vida comunitaria y relaciones interpersonales, mientras que el SDQ se enfoca principalmente hacia las relaciones interpersonales, la conducta y el comportamiento del niño.

En términos generales y frente a los resultados psicométricos positivos presentados anteriormente, se concluye que los padres y los profesores brindan información adecuada a través de la técnica de entrevista, la cual es fiable para identificar consecuencias en el funcionamiento de niños y niñas con TDAH $(20,21)$.

Los cuestionarios CLARP pueden ser aplicados por diferentes profesionales y por el personal que interviene a los niños y niñas con TDAH, entre ellos: médicos, psicólogos, terapeutas ocupacionales, fisioterapeutas, fonoaudiólogos, educadores especiales y docentes. En tal sentido, favorece la intervención interdisciplinaria e integral a los niños y niñas en busca de su proceso de rehabilitación; además permite evidenciar cambios en el curso del tiempo de evolución del trastorno. Además es de reconocer que los cuestionarios son de fácil y clara aplicación, y requieren mínimo entrenamiento desde las bases conceptuales y la aplicación de la CIF (22).

Los cuestionarios CLARP-TDAH padres y profesores son confiables desde su consistencia interna y el CLARP-TDAH profesores es efectivo desde su validez concurrente para su aplicación; por lo tanto, este último tiene mayores fortalezas psicométricas de validez y de confiabilidad, reconociendo al igual que otros autores como Loe y Feldman que su aplicación permitiría determinar puntualmente los problemas en el contexto escolar (23).

Los cuestionarios permiten identificar problemas y dificultades específicas y relevantes, y facilitar el diseño de un plan más apropiado de intervención desde las diversas disciplinas de apoyo terapéutico que intervienen a los niños y las niñas con TDAH, esto al mismo tiempo logra apoyar la utilidad de la CIF, en cuanto a 
aportar a un lenguaje común, estandarizado, fiable y aplicable transdisciplinar y transculturalmente con el fin de describir determinantes de funcionamiento en esta población $(24,25)$.

\section{Conclusiones}

El cuestionario CLARP-TDAH profesores posee adecuadas propiedades psicométricas, por lo tanto se puede iniciar su uso y aplicación clínica. El cuestionario CLARP-TDAH padres aun no arroja propiedades psicométricas fuertes que permitan su uso de manera rigurosa, válida y confiable. En niños y niñas con TDAH es necesario conocer información adicional que vaya más allá del diagnóstico, es importante separar los signos y síntomas de las consecuencias para un mejor entendimiento del trastorno, por lo tanto, se deben tener en cuenta otras alteraciones funcionales que trasciendan a la observación clínica en los diferentes ámbitos familiar y escolar y desde las conductas y comportamientos adaptativos personales y sociales. La CIF tiene como propósito general facilitar un trabajo interdisciplinario y un lenguaje estándar para describir los resultados de salud corporal en relación a sus funciones y estructuras, de la persona en el desempeño de sus actividades cotidianas y en su participación en sociedad, por lo tanto, se constituye en una herramienta que facilita un abordaje integral a los niños con TDAH. Además, los resultados de su aplicación pueden aportar de manera relevante para la toma de decisiones desde la política pública para la infancia. El CLARP-TDAH padres y el CLARP-TDAH profesores se constituye en una herramienta que favorece la intervención interdisciplinaria e integral a los niños en busca de su proceso de rehabilitación, además que permite evidenciar cambios en el curso del tiempo de evolución del trastorno.

\section{Descargos de responsabilidad}

Los autores manifiestan que el presente estudio recibió financiación de la Universidad Autónoma de Manizales y no declaran conflictos de interés.

\section{Bibliografía}

1. American Academy of Pediatrics. Subcommittee on Attention-Deficit/Hyperactivity Disorder, Steering Committee on Quality Improvement and Management. ADHD: Clinical Practice Guideline for the Diagnosis, evaluation and treatment of attention deficit/hyperactivity disorder in children and adolescents. Pediatrics. 2011;128:1007-22.

2. American Academy of Pediatrics. Commitee on Quality Improvement and Subcommitte on AttentionDeficit/Hyperactivity Disorder. Clinical Practice Guideline: Diagnosis and evaluation of the child with attention-deficit/hyperactivity disorder. Pediatrics. 2001;105:1158-70.

3. García-Pérez A, Expósito-Torrejón J, Martínez-Granero MA, Quintanar-Rioja A, Bonet-Serra B. Semiología clínica del trastorno por déficit de atención con hiperactividad en función de la edad y eficacia de los tratamientos en las distintas edades. Rev Neurol. 2005;41:517-24.

4. Cornejo JW, Osío O, Sánchez Y, Carrizosa J, Sánchez G, Grisales H, et al. Prevalencia del trastorno por déficit de atención-hiperactividad en niños y adolescentes colombianos. Rev Neurol. 2005;40:716-22.

5. Roselló B, Amado L, Bo RM. Patrones de comorbilidad en los distintos subtipos de niños con trastorno por déficit de atención con hiperactividad. Rev Neurol Clin. 2000;1:181-92.

6. World Health Organization. The tenth revision of the international classification of diseases and related health problems. Geneve: World Health Organization; 1992. 
7. American Psychiatric Association. Diagnostic and Statistical Manual of Mental Disorders. $4^{\text {th }}$ ed. Washington, DC: American Psychiatric Association; 1994.

8. World Health Organization. International classification of functioning, disability and health. Geneva: World Health Organization; 2001.

9. Salamanca, LM. Construcción, validación y confiabilidad de un cuestionario sobre niños y niñas con TDAH. Rev Latinoam Cienc Soc Niñez Juv. 2010:8(2):1117-29.

10. Salamanca LM. Cuestionario para la evaluación de limitaciones en la actividad y restricciones en la participación en niños con TDAH a nivel escolar. Rev Chil Psiquiatr Neurol Infanc Adolesc. 2010: 21(1):19-30.

11. Goodman R. The strengths and difficulties questionnaire: a research note. J Child Psychol Psychiatry. 1997:38(5):581-6.

12. Goodman R, Ford T, Simmons H, Gatward R, Meltzer H. Using the Strengths and Difficulties Questionnaire (SDQ) to screen for child psychiatric disorders in a community sample. Br J Psychiatry. 2000:177:534-9.

13. Stone LL, Otten R, Engels RC, Vermulst AA, Janssens JM. Psychometric properties of the parent and teacher versions of the strengths and difficulties questionnaire for 4-to-12-year-olds: A review. Clin Child Fam Psychol Rev. 2010;13(3):254-74.

14. Russell G., Rodgers LR, Ford T. The strengths and difficulties questionnaire as a predictor of parentreported diagnosis of autism spectrum disorder and attention deficit hyperactivity disorder. PloS One. 2013:8(12):e80247.

15. Ustün TB. Using the international classification of functioning, disease and health in attention-deficit/ hyperactivity disorder: separating the disease from its epiphenomena. Ambul Pediatr. 2007;7(1 Suppl):132-9.

16. Bölte S, De Schipper E, Holtmann M, Karande S, de Vries PJ, Selb M, et al. Development of ICF Core Sets to standardize assessment of functioning and impairment in ADHD: the path ahead. Eur Child Adolesc Psychiatry. 2013:1-10.

17. Cortina JM. What is coefficient alpha? An examination of theory and applications. J Applied Psycho. 1993;78(1):98-104.

18. Pineda DA, Henao GC, Puerta IC, Mejía SE, Gómez LF, Miranda ML, et al. Uso de un cuestionario breve para el diagnóstico de deficiencia atencional. Rev Neurol. 1999;28(4):365-72.

19. Battaglia M, Russo E, Bolla A, Chiusso A, Bertelli S, Pellegri A, et al. International classification of functioning, disability and health in a cohort of children with cognitive, motor, and complex disabilities. Dev Med Child Neurol. 2004;46(2):98-106.

20. Ortiz JA, Acle G. Diferencias entre padres y maestros en la identificación de síntomas del trastorno por déficit de atención con hiperactividad en niños mexicanos. Rev Neurol. 2006;42(1):17-21.

21. Cardo E, Servera M, Vidal C, De Azua B, Redondo M, Riutort L. Influencia de los diferentes criterios diagnósticos y la cultura en la prevalencia del trastorno por déficit de atención/hiperactividad. Rev Neurol. 2011;52(supl 1):s109-17.

22. Kronk RA, Ogonowski JA, Rice CN, Feldman HM. Reliability in assigning ICF codes to children with special health care needs using a developmentally structured interview. Disabil Rehabil. 2005;27(17):977-83.

23. Loe IM, Feldman HM. Academic and educational outcomes of children with ADHD: literature review and proposal for future research. Ambul Pediatr. 2007;7(1):82-90.

24. Steiner WA, Ryser L, Huber E, Uebelhart D, Aeschlimann A, Stucki G. Use of the ICF model as a clinical problem-solving tool in physical therapy and rehabilitation medicine. Phys Ther. 2002;82(11):1098-107

25. Mahone, EM, Schneider HE. Assessment of attention in preschoolers. Neuropsychol Rev. 2012;22(4):361-83. 